Check for updates

Cite this: Mater. Horiz., 2018, 5,86

Received 29th June 2017

Accepted 4th December 2017

DOI: $10.1039 / \mathrm{c} 7 \mathrm{mh} 00486 a$

rsc.li/materials-horizons

\section{Accelerated atomic-scale exploration of phase evolution in compositionally complex materials $\dagger$}

\author{
Y. J. Li, (D) ${ }^{a}$ A. Savan, (D) ${ }^{b}$ A. Kostka, (D) ${ }^{a}$ H. S. Stein $\left(\mathbb{D}^{b}\right.$ and A. Ludwig (D) *ab
}

\begin{abstract}
Combining nanoscale-tip arrays with combinatorial thin film deposition and processing as well as direct atomic-scale characterization (APT and TEM) enables accelerated exploration of the temperature- and environment-dependent phase evolution in multinary materials systems. Results from nanocrystalline $\mathrm{CrMnFeCoNi}$ show that this alloy is unstable and already decomposes after $1 \mathrm{~h}$ at low temperatures of around $300{ }^{\circ} \mathrm{C}$. The combinatorial processing platform approach is extendible to explore oxidation and corrosion in complex structural and functional materials on the atomic scale.
\end{abstract}

Compositional complexity is necessary for advanced materials to fulfil the challenging and frequently contradictory requirements of technical applications. ${ }^{1-3}$ The indispensability of multiple elements to achieve multifunctionality leads to alloys consisting of 5 to 12 elements: e.g. steels, glasses, superalloys, and new compositionally complex materials (CCM), the so-called "high-entropy alloys" and "multiple principal element alloys" (MPEAs). The number of possible CCMs is vast, and e.g. MPEAs are largely unexplored, promising materials discoveries. However, the exploration and design of new materials requires understanding of the atomicscale details of phase evolution (phase formation, stability ranges, and decomposition pathways) in dependence on processing conditions (temperature, time, environment). The ability to discover and track the formation and evolution of all possible phases, from an initial state of a multinary system via nucleation and growth upon thermal annealing or reaction, is of fundamental importance in materials science. Whereas the ground state stability of new compounds can be predicted by highthroughput $a b$ initio methods, ${ }^{4}$ this does not yet extend to the phase formation and stability of CCMs at elevated temperatures. This makes efficient and systematic experiments necessary. Combinatorial materials science ${ }^{5}$ accelerated the discovery and

\footnotetext{
${ }^{a}$ ZGH, Ruhr-University Bochum, Bochum, Germany.E-mail: alfred.ludwig@rub.de

${ }^{b}$ Institute for Materials, Ruhr-University Bochum, Bochum, Germany

$\dagger$ Electronic supplementary information (ESI) available: Methods, extended results including additional table, figures and movie, and any associated references. See DOI: $10.1039 / \mathrm{c} 7 \mathrm{mh} 00486 \mathrm{a}$
}

\begin{abstract}
Conceptual insights
Single-phase compositional complex materials (CCM) show potential for applications due to their unusual mechanical properties. However, they may decompose into multiple phases at elevated temperatures or reactive environments such that their superior properties disappear, causing potentially catastrophic failure. The challenge is to investigate their phase stability in an accelerated way, since the existing approach, mainly consisting of casting, long-term annealing and preparation of samples for microscopy, frequently requires months or even years. Our concept is to replace all processes by a new fast combinatorial processing platform approach, which enables: simultaneous synthesis of 36 identical atomic-scale-mixed films by co-deposition of 5 elements on an array of $10 \mathrm{~nm}$-diameter Si tips; rapid phase evolution in the formed nanoscale reactors upon processing; direct atomicscale analysis of phase evolution after each processing step by atom probe tomography, complemented by transmission electron microscopy. Using this approach, we efficiently elucidate phase evolution by monitoring the redistribution of individual atoms upon annealing. This concept will accelerate the design of novel structural and functional nanocrystalline multinary materials with superior properties, as the complete phase space of multiple principal element alloys can be quickly explored, resulting in the knowledge of which phases form or decompose at different processing conditions.
\end{abstract}

targeted development of new materials, and its use for identification and optimization of MPEAs was proposed. ${ }^{6}$ However, possibilities to accelerate understanding of complex alloys by combining combinatorial synthesis of materials and direct atomic-scale characterization of their thermal and chemical stability have not been addressed.

We present a new approach to accelerate atomic-scale phase evolution studies in complex materials: a combinatorial processing platform (CPP) consisting of parallelly synthesized and processed, and thus identical, nanoscale volumes $(\sim 100 \mathrm{~nm}$ length, about $10^{8}$ atoms) of the material of interest, which serve as "fast-reaction chambers", ready for direct atom probe tomography (APT) analysis, and suitable for correlative transmission electron microscopy (TEM) studies (Fig. 1). Key to the CPP approach is, that one identical, highly uniform and atomicscale mixed multinary film on all tips is processed sequentially 
a)
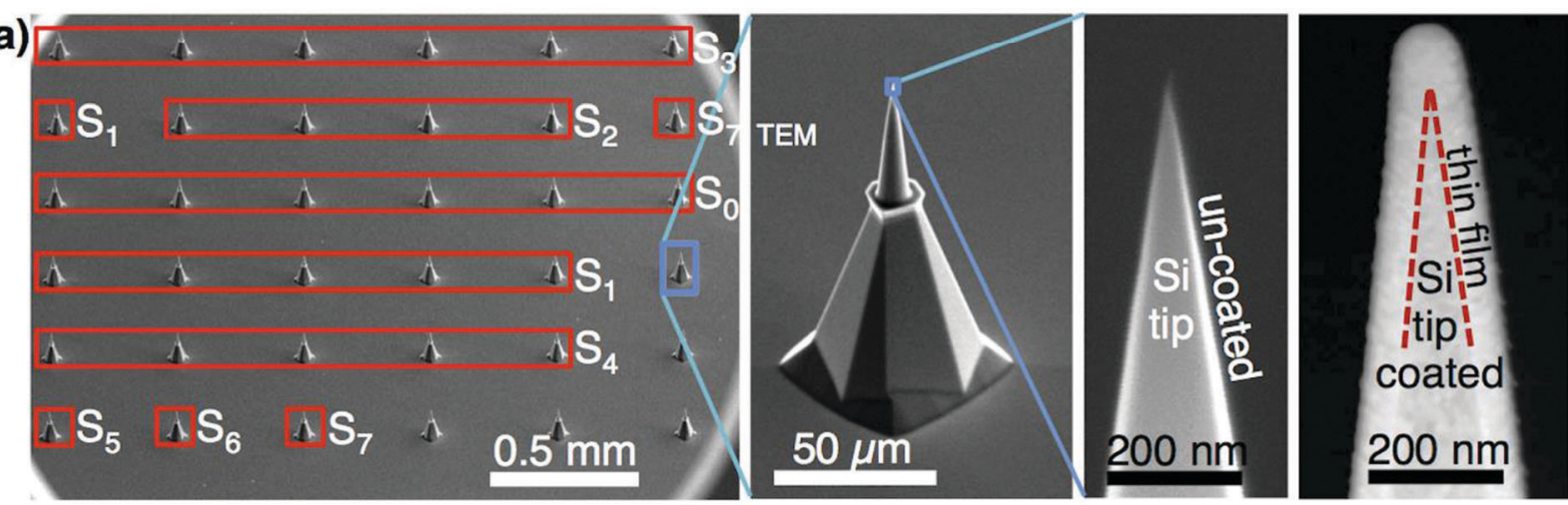

b)

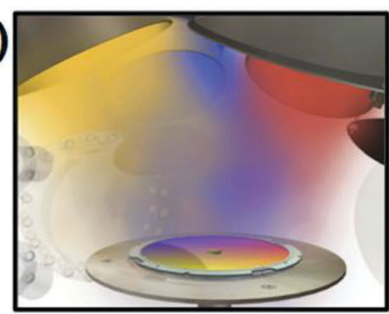

c)

annealing
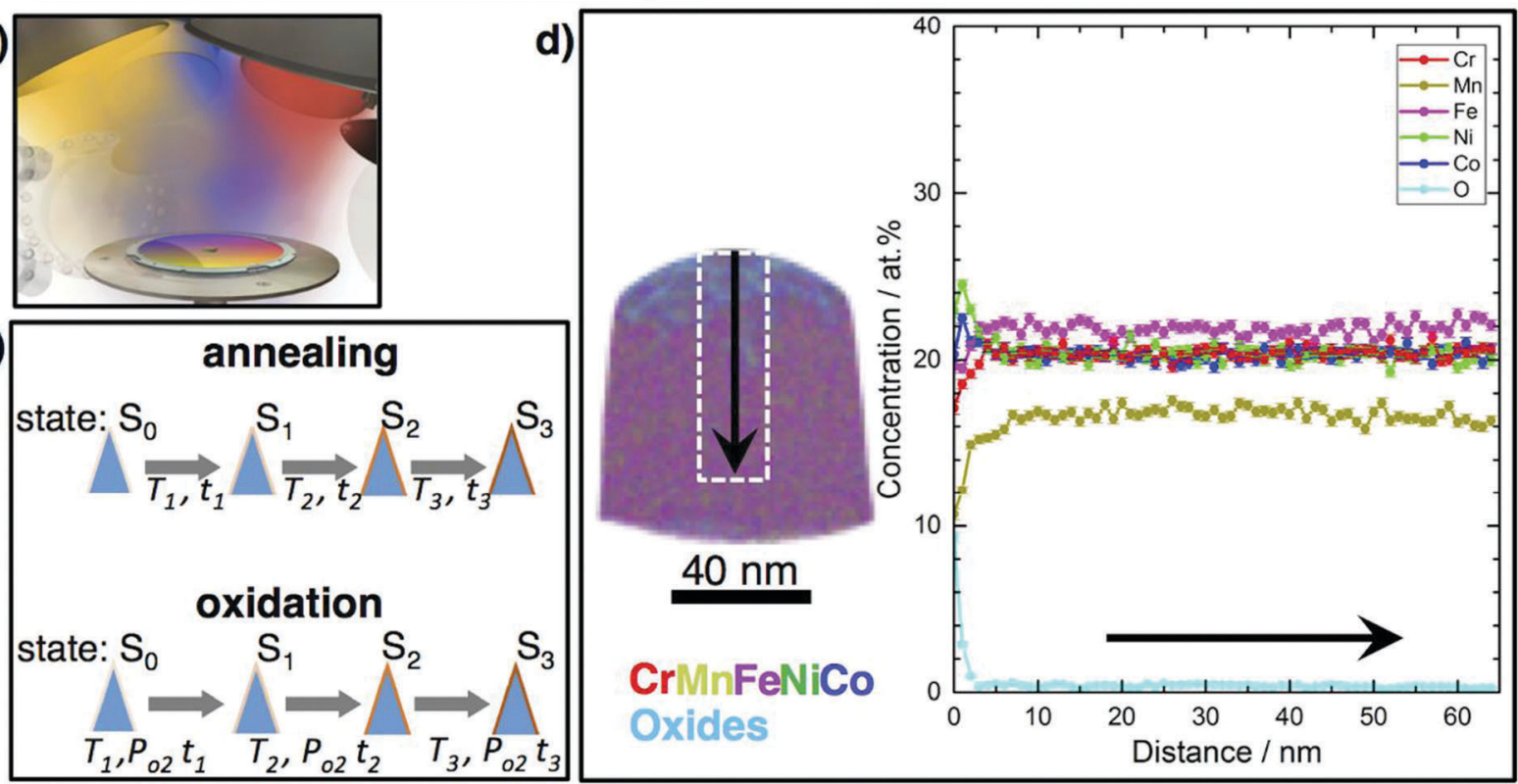

Fig. 1 Combinatorial processing platform (CPP) for atomic-scale analysis of phase evolution in nanoscale multinary thin films. (a) SEM image of a Si tip array with 36 tips. Boxes indicate which tips were used for measurements in each processing state $\mathrm{S}_{x}$. Middle: Magnified SEM image of an uncoated tip and its sharp end which serves as substrate for the combinatorial deposition of the multinary film. Right: TEM image of a CrMnNiCoFe coated and annealed tip is shown $\left(350^{\circ} \mathrm{C}, \mathrm{S}_{7, T E M}\right.$ ). (b) Schematic of the co-deposition process as applied on a CPP and other substrates (wafer, single APT tips and TEM-membrane chips): five sputter cathodes deposit simultaneously an atomically mixed film (rotating substrate: homogeneous film; fixed substrate: composition spread). (c) Examples of combinatorial processing using the CPP (a tip is indicated by a blue triangle, the nanoscale coating with orange colors). (d) Example APT result of a CrMnNiCoFe film in state $S_{3}\left(150{ }^{\circ} \mathrm{C}, 1 \mathrm{~h}\right)$. The dashed rectangle marks the region of concentration analysis, while arrows show the direction of the concentration profiles. A $\sim 3 \mathrm{~nm}$ thick oxide layer is observed on the film surface, see O-concentration profile (bright blue line).

at different conditions. The nanoscale film volume on a tip is sufficiently large to avoid "nano-effects" or influences by the tip curvature $^{7}$ on phase evolution. Therefore, results obtained from the expedited nanoscale metallurgy on tips can be used generally for identification of all (decomposition) phases in arbitrary multinary bulk systems. The resulting chemical distributions, phase formation and evolution are directly tracked, for up to 36 APT measurements on different processing states. Timeconsuming procedures (casting, long-term annealing of bulk materials, sample preparation for atomic-scale microscopies) are not necessary. The CPP approach is fast, clean and structural damage-free, as no focused ion beam (FIB) preparation is necessary. All samples are batch-processed, saving time, maintaining uniformity and ensuring direct comparability. For each processing state $\mathrm{S}_{x}$, several coated tips can be consumed: Fig. 1a shows the tips measured at each stage of this study. Ideally, phase-evolution tracking starts from a perfectly mixed initial state of a multinary system, which can be best achieved by a co-deposited film, where all atoms condense randomly. The films' "fast-reaction chambers" allow rapid (several hours) and thorough diffusion as necessary diffusion lengths for achieving equilibrium are only 10's of nm. New phases will form, unless the initial mixture is already thermodynamically (meta)stable.

The CPP is realized via direct combinatorial co-sputter deposition from high-purity elemental targets on a commercially available array of 36 pre-sharpened Si tips (Fig. 1a). During deposition, the tip array rotates to achieve a conformal layer with the highest possible mixing of the multinary film (Fig. 1b). The coated tip array serves as a CPP, enabling direct atomic-scale characterization using APT and TEM, for different 
processing states, $\mathrm{S}_{x}$, of the identical material (Fig. 1c). Physical vapor deposition methods like electron-beam evaporation, ionbeam- and magnetron-sputtering were used to coat individual tips, e.g. single electropolished W-, and Ni-APT tips, with layer stacks, e.g. $\mathrm{Ni} / \mathrm{Al}$ or $\mathrm{NiFe} / \mathrm{Cu}$ for the investigation of atomic structure and reactions at interfaces, e.g. upon annealing up to $500{ }^{\circ} \mathrm{C}$ for $40 \mathrm{~min} .{ }^{8,9}$ However, as APT is destructive, we introduce co-deposition from several sources on CPP (Fig. 1b), which overcomes the limitations of relying on individual tips. This enables systematic processing investigations up to temperatures of at least $350{ }^{\circ} \mathrm{C}$ (Fig. 1c) for annealing on the order of hours, and $500{ }^{\circ} \mathrm{C}$ for annealing on the order of minutes (Fig. 5).

APT determines the actual 3D distribution of the atomic species in detail at the atomic scale with high compositional accuracy and statistical significance, ideal to study phase evolution of CCMs. In correlation with TEM, e.g. by FIB-transfer of a coated tip from the CPP to a TEM holder, the compositional phase evolution is confirmed by energy-dispersive X-ray analysis (EDX), whereas the structural phase evolution is confirmed by imaging and electron diffraction. In combination, the phase evolution on the atomic scale is monitored effectively. However, the analysis of this high-dimensional data requires new visualization approaches, described below.

Thus, CPPs expedite the systematic and high quality atomicscale data acquisition, as a basis for observing and understanding phase evolution (emerging phases and possible decomposition pathways) in dependence on processing conditions.

We demonstrate the feasibility of the CPP approach by efficiently establishing the phase constitution of a quinary CCM for different temperatures and annealing environments (vacuum, air; see ESI $\dagger$ for details) and comparing the obtained results to literature. As a proof of principle system for expedited nanoscale metallurgy $\mathrm{CrMnFeCoNi}^{10}$ is taken, hereafter this equiatomic face-centered cubic (fcc) single phase solid solution is denoted as "Cantor phase". While this material has excellent toughness at and below room temperature and simultaneous increase of strength and ductility with decreasing temperature down to $-196{ }^{\circ} \mathrm{C},{ }^{3}$ it shows limited thermal and compositional stability at elevated temperatures. Bulk samples (grain size $1 \mathrm{~mm}$ ) decompose at $500{ }^{\circ} \mathrm{C}$ after annealing for 500 days into the phases $\mathrm{L}_{1}$-NiMn, B2-FeCo and a $\mathrm{Cr}$-rich body-centered cubic (bcc) phase. A Cr-rich $\sigma$ phase forms after 500 days at $700{ }^{\circ} \mathrm{C} .{ }^{11}$ To achieve these results in conventional coarsegrained materials, such extremely long annealing times are necessary, precluding the high-throughput exploration of the vast MPEA composition space. Recently, nanocrystalline (nc) Cantor phase samples (grain size $50 \mathrm{~nm}$ ) were prepared by severe plastic deformation (SPD) using high pressure torsion (HPT). Decomposition into the phases $\mathrm{L}_{0}$-NiMn and $\mathrm{Cr}$-rich bcc occurred already after 5 min upon annealing at $450{ }^{\circ} \mathrm{C}$; after $15 \mathrm{~h}$ the B2-FeCo phase formed. ${ }^{12}$ Thus, SPD offers a possibility to trigger rapid phase decomposition, but it is challenging to do and not suitable for all materials. It is unclear if the low stability of the SPD Cantor phase is due to its nanoscale structure (short diffusion paths, enhanced grain boundary diffusivity) or to effects of the SPD treatment, or both.
In comparison to SPD approaches sputter deposition can synthesize nc samples of even finer grains without strong modification of grain boundary properties. Following the present approach, the tip array was conformally coated with CrMnFeNiCo by combinatorial co-sputtering, without damage to the tips, thus forming a CPP. TEM on a simultaneously coated $\mathrm{Cu}-\mathrm{C}$ grid shows that the as-deposited film $\left(\mathrm{S}_{0}\right)$ is a nanocrystalline single phase fcc solid solution (Fig. S2, ESI $\dagger$ ). APT of the $S_{0}$ film is difficult due to rapid fracture under the applied high electric field, and could only be performed in laser mode for short times. We relate this to the non-continuous nanostructure of the $\mathrm{S}_{0}$ film. Temperature-dependent resistivity measurements (not shown) indicate that the film densifies upon heating from $\mathrm{S}_{0}$. One coated $\mathrm{CPP}$ was annealed in vacuum at temperatures from $100{ }^{\circ} \mathrm{C}$ to $350{ }^{\circ} \mathrm{C}$ (holding time $1 \mathrm{~h}$ at each temperature $>100{ }^{\circ} \mathrm{C}$, total thermal processing time $7.5 \mathrm{~h}$; for processing details see Table S1, Fig. S1 and S3, ESI $\dagger$ ) and was characterized in each state to identify the temperature range where decompositions occur. Up to at least $300{ }^{\circ} \mathrm{C}$ the Si tip does not react with the coating. Interdiffusion does not occur, enabling the study of the film properties alone (Fig. S4, ESI $\dagger$ ). The native Si-oxide may serve as a diffusion barrier. At $350{ }^{\circ} \mathrm{C}$ Mn diffuses into Si. However, the nanoscale reactor volume of the film remains without $\mathrm{Si}$ thus still allowing the phase evolution study up to $350{ }^{\circ} \mathrm{C}$, see below. Future applications might require an additional diffusion barrier, e.g. by atomic layer deposition. Coated tips from all $\mathrm{S}_{x}$ could be measured by APT, with up to $>50$ million ions collected from one tip.

Fig. 1d shows exemplary APT results of the CPP in state $\mathrm{S}_{3}$ $\left(150{ }^{\circ} \mathrm{C}, 1 \mathrm{~h}\right)$ : whereas the $\mathrm{S}_{0}$ film is equiatomic, a Mn-loss upon annealing of 4 at\% occurs, due to the high vapor pressure of $\mathrm{Mn}$. By increasing the $\mathrm{Mn}$ content in $\mathrm{S}_{0}$, the equiatomic composition in the film volume can be maintained to higher temperatures. As the Cantor phase shows a large compositional existence range, the study was continued with the nonequiatomic composition. Fig. 1d shows that the surface of the film contains a Mn-rich cap followed by a few nm thick layer of mainly Cr- and Mn-oxides which were confirmed by TEM electron diffraction data for annealing above $150{ }^{\circ} \mathrm{C}$ (Table S2, ESI $\dagger$ ). The film volume remains almost equiatomic except for Mn. Fig. 2 shows atom maps for all elements in states $S_{1}$ to $S_{7}$ (see Fig. S3 and Table S1, ESI $\dagger$ ). After annealing at $100{ }^{\circ} \mathrm{C}$ for $0.5 \mathrm{~h}\left(\mathrm{~S}_{1}\right)$, Ni and $\mathrm{Mn}$ segregate to grain boundaries, revealing also the nanostructure of the Cantor phase film. This segregation increases with annealing time and temperature up to $S_{5}$. The distribution of $\mathrm{Fe}, \mathrm{Co}$, and $\mathrm{Cr}$ remains homogeneous, except for a few clusters containing all elements at grain boundary triple junctions. A grain size of $\sim 10 \mathrm{~nm}$ is deduced from this analysis, consistent with TEM of an identical film on a flat substrate (Fig. S2, ESI $\dagger$ ). Neither APT nor TEM indicate grain growth, up to $\mathrm{S}_{5}\left(250^{\circ} \mathrm{C}\right)$. In state $\mathrm{S}_{6}$ and $\mathrm{S}_{7}$ clear compositional zones in the film are observed, which are enriched or depleted by various combinations of all elements. A binomial frequency distribution analysis was performed, Fig. S5 (ESI $\dagger$ ), quantifying the (in)homogeneity of the elements in the film at different states. 


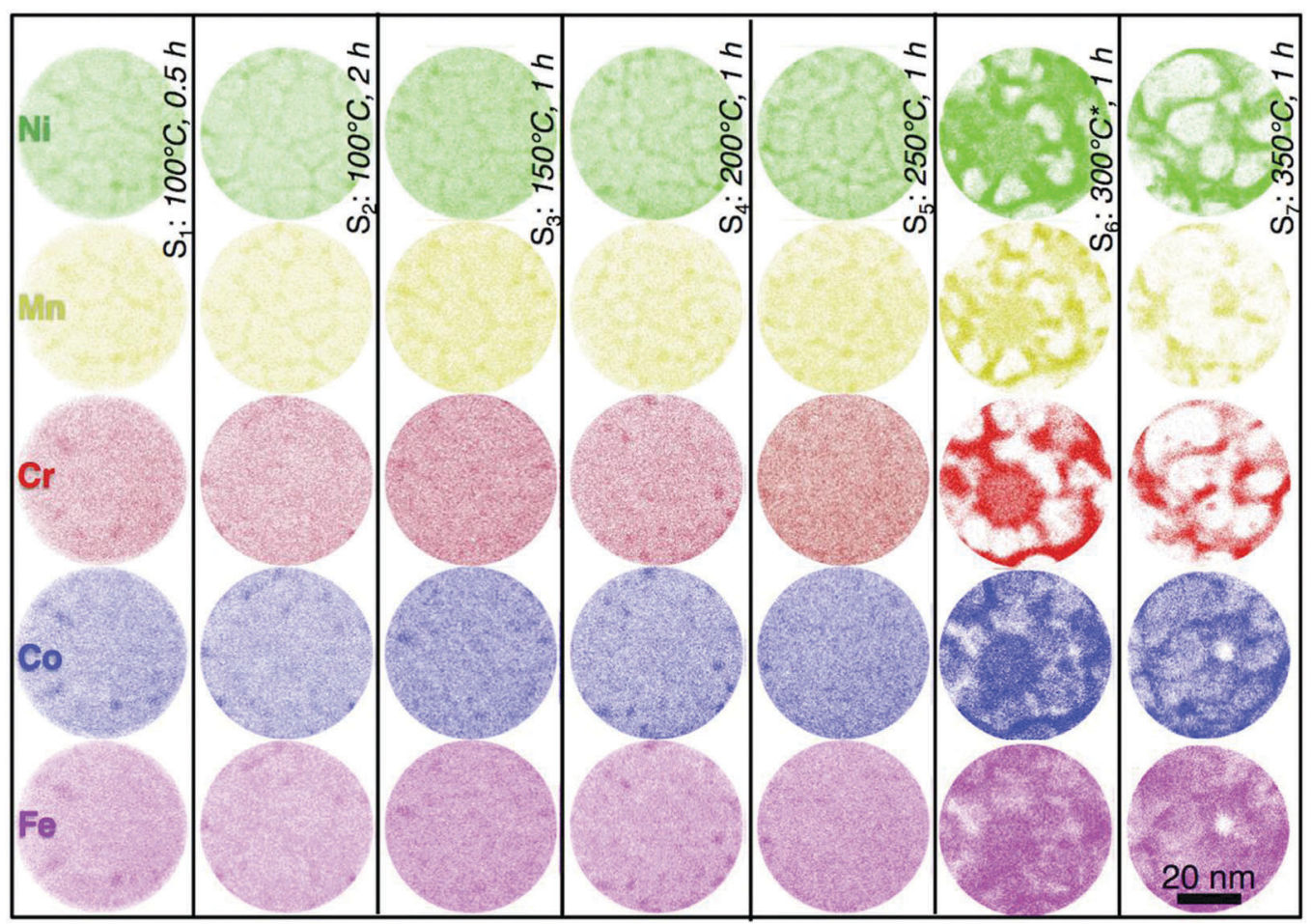

Fig. 2 Atomic-scale constitution of the CrMnFeCoNi film on CPP in subsequent thermal processing states. Cross-sectional APT element-distribution maps for $\mathrm{Ni}$ (green), Mn (yellow), $\mathrm{Cr}$ (red), $\mathrm{Co}$ (blue), and Fe (pink) show homogeneous distribution of all elements in the nanoscale grain interiors and segregation of $\mathrm{Ni}$ and $\mathrm{Mn}$ at grain boundaries of the Cantor phase up to $\mathrm{S}_{5}$. Decomposition of the Cantor phase starts at $\mathrm{S}_{6}$.

Fig. 3 shows the atomic-scale constitution of the film in longitudinal sections in states $\mathrm{S}_{6}$ (Fig. $3 \mathrm{a}$ and $\mathrm{b}$ ) and $\mathrm{S}_{7}$ (Fig. 3c and $\mathrm{d}$ ). For a clear visualization of different phases after decomposition of the Cantor phase, only $\mathrm{Cr}$ and $\mathrm{Ni}$ atoms are displayed in the first atom map. The Ni distribution along with isoconcentration surfaces plotted at 40 at $\% \mathrm{Cr}, 40$ at $\% \mathrm{Fe}$ and 30 at $\%$ Co are shown in the second atom map. The concentration profiles derived from two regions of interest (ROI) indicate that the film decomposes into four additional phases next to a remaining volume fraction of the Cantor phase: a FeCo-rich, a highly Cr-rich (>70 at\%), a Cr-rich $\left(\sim 40\right.$ at\%), and a Ni-rich phase. In state $S_{7}$ the Cantor phase is completely decomposed into the phases already observed at $\mathrm{S}_{6}$, with slight changes in their chemical composition (see Table S1, ESI $\dagger$ ). The crystal structures of these phases are identified by complementary TEM investigations (Fig. S6, ESI $\dagger$ ) as FeCo-B2, Cr-rich (> 70 at\% Cr) bcc, Cr-rich ( $\sim 46$ at\% $\mathrm{Cr}) \sigma$, and NiMn-rich $\mathrm{L} 1_{0}$. The decomposition process is summarized as follows: $>300{ }^{\circ} \mathrm{C} \mathrm{Ni}$ and $\mathrm{Mn}$ diffuse into grain boundaries, leaving grain interiors nearly $\mathrm{Ni}$ - and $\mathrm{Mn}$-free. The $\mathrm{L} 1_{0}$ phase occupies the major volume of the grain boundaries. A Cr-rich phase is adjacent to the $\mathrm{L} 1_{0}$ phase. Fe and Co remain mainly in grain interiors and form a $\mathrm{B} 2$ phase surrounded by the $\mathrm{L} 1_{0}$ phase. Such atomic-scale insights expand our understanding of the phase decomposition process.

Using FIB, a coated and fully thermally processed $\left(\mathrm{S}_{7}\right)$ tip from the CPP was transferred to TEM for further analysis. Fig. 4a reveals the nanoscale structure of the CrMnNiCoFe film in this state. The sequentially annealed film is still conformally coated on the Si tip, which is clearly visible and separated from the film by a still-sharp interface. The morphology of the tip and the thickness of the coated film in TEM are nearly identical to those from APT analysis. Furthermore, the phase decomposition in state $S_{7}$ is documented on the electron diffraction pattern (Fig. 4b and Table S2, ESI $\dagger$ ), consistent with APT results (Fig. 3d).

These results confirm that the film on the tip forms a volume where fast diffusion and reaction occurs. This ensures that for short annealing times sufficient diffusion takes place such that all phases out of the multinary film will form. The CPP approach thus accelerates the study of the phase stability of MPEAs and reveals important details of the decomposition pathways. It is worth noting that the limit of the thermal stability in terms of annealing temperature and time obtained in the nano-sized samples may not be directly applied to their conventional coarse-grained bulk counterparts due to the difference in thermodynamics and kinetics of decomposition process related to diffusion paths, diffusivities, and grain boundary volume and energies. Due to the fact that the nanosized samples yield the same phase constituents as the bulk materials, the current approach can be applied to efficiently evaluate and select the most appealing alloy system and compositions from among many candidates. For potential industrial application the selected alloy needs to be further studied following the traditional metallurgical and characterization procedures. Therefore, the value of this novel approach is not to replace the 

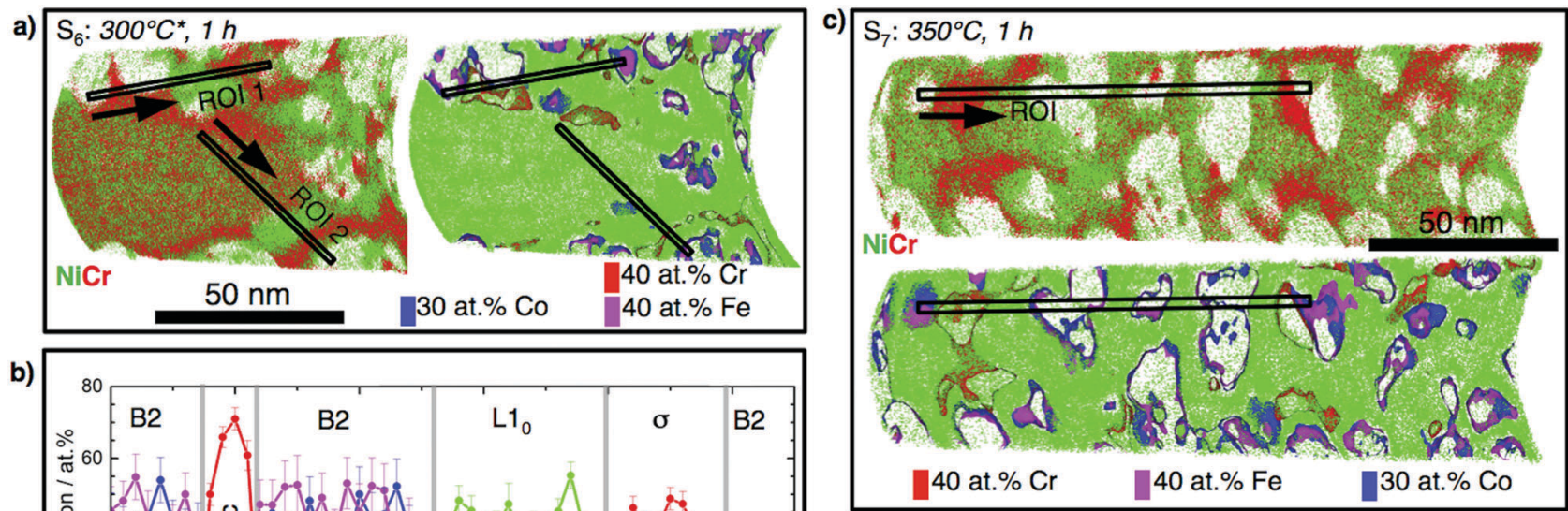

b)
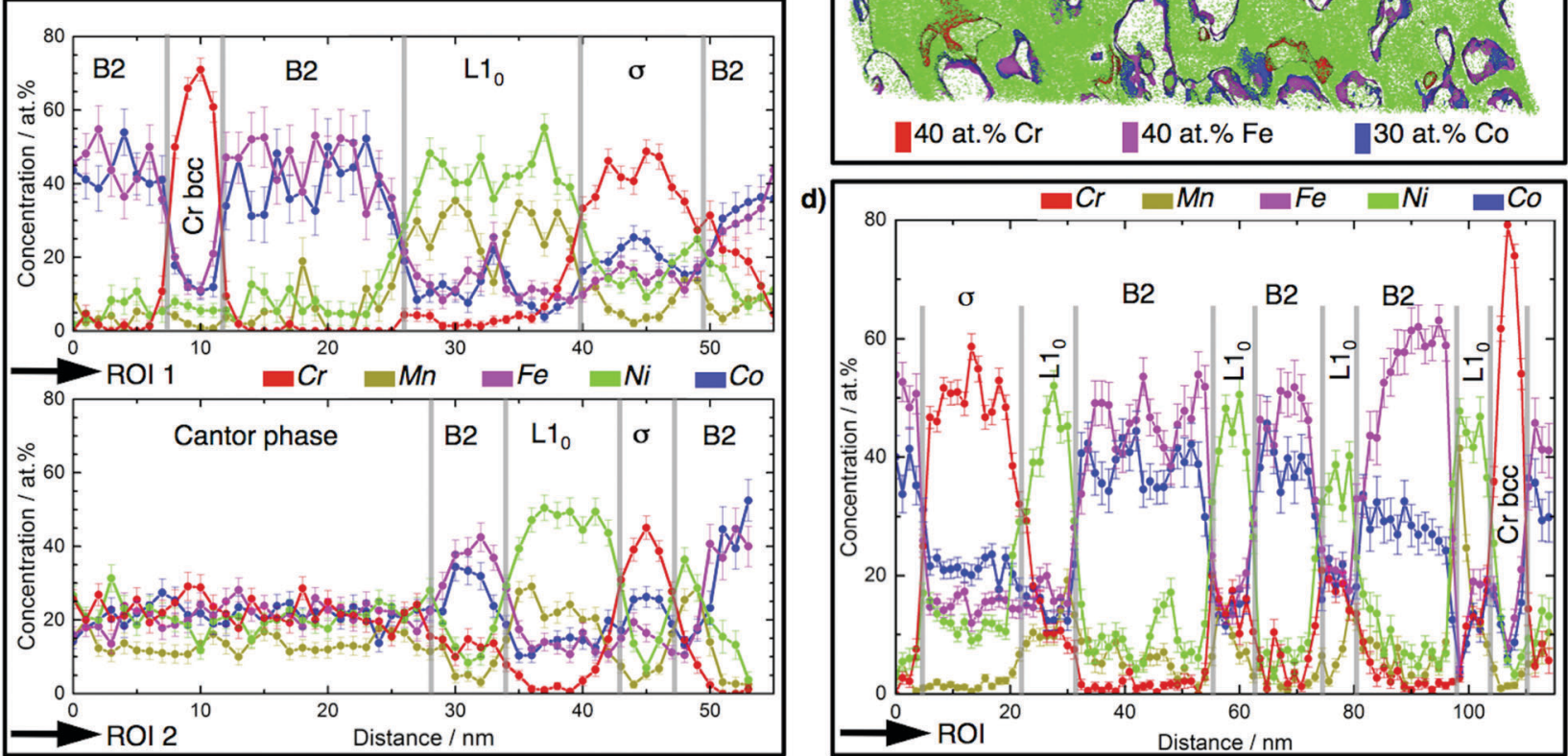

Fig. 3 Atomic-scale constitution of partially $\left(\mathrm{S}_{6}\right)$ and completely $\left(\mathrm{S}_{7}\right)$ decomposed CrMnFeCoNi films into the phases B2 (FeCo-rich), $\sigma$ (40 at\% Cr), $\mathrm{Cr}$-bcc ( $>70$ at\% $\mathrm{Cr}$ ), and $\mathrm{L1}_{0}$ (NiMn-rich). (a) State $\mathrm{S}_{6}$. Left: Atom maps of $\mathrm{Ni}$ and $\mathrm{Cr}$. Right: Ni map along with the isoconcentration surfaces plotted at 40 at\% $\mathrm{Cr}$ (red), 40 at\% Fe (pink), and 30 at\% Co (red) which highlight the Cr-rich bcc, $\sigma$ and FeCo-rich B2 phases. (b) 1-D concentration profiles for the selected ROIs shown in (a). ( $c$ and d) Analogous to ( $a$ and b) for $S_{7}$. Arrows mark the directions along which 1-D concentration profiles are plotted. For atom maps of other elements see Fig. S7, ESI. $\dagger$
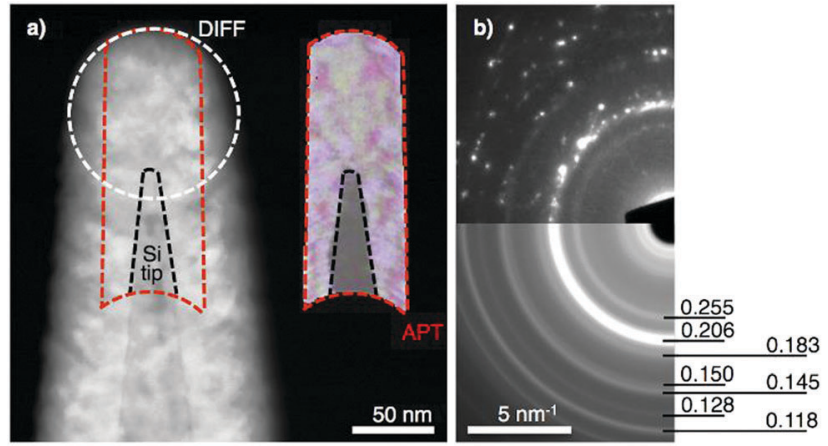

Fig. 4 TEM of a CrMnFeCoNi film on a Si tip in state $\mathrm{S}_{7}\left(350^{\circ} \mathrm{C}\right)$. (a) High angle annular dark field image showing the tip and the grain structure of the film $\left(\mathrm{S}_{7}, \mathrm{TEM}\right)$ in comparison to an APT analysis from a tip from the same CPP and state $\left(S_{7}\right)$. (b) Electron diffraction acquired from the encircled region in (a) and its rotational averaging with measured interplanar distances in $\mathrm{nm}$.

traditional procedure, but to strongly reduce its related cost and time requirements. In comparison to coarse-grained material with grain size of $1 \mathrm{~mm},{ }^{11}$ in which decomposition occurs at $500{ }^{\circ} \mathrm{C}$ after 500 days, the time needed for the decomposition in the nc film is four orders of magnitude lower. Considering the grain size being about five orders of magnitude lower than that of the coarsegrained material, the rapid decomposition process can be mainly attributed to the features of the sputtered nc samples, such as short diffusion paths and enhanced diffusivities. It is a general view that diffusion in nanocrystalline solids occurs along grain boundaries due to their large volume fraction, which is similar or even faster than normal grain boundary diffusion, ${ }^{13-16}$ resulting in the formation of stable or metastable phases at lower temperatures without kinetic limitations. The current approach confirms this and successfully triggers phase separation at $300{ }^{\circ} \mathrm{C}$ in $1 \mathrm{~h}$ for CrMnFeNiCo. The HPTed nc samples also show early phase separation at $450{ }^{\circ} \mathrm{C}$ after $5 \mathrm{~min},{ }^{12}$ however this very fast decomposition may involve additional mechanisms which are related to HPT. HPT introduces a high density of defects such as dislocations (up to $10^{16} \mathrm{~m}^{-2}$ ) and vacancies into materials, which yields non-equilibrium grain boundaries together with enhanced diffusivity ${ }^{13,14}$ facilitating fast grain growth and decomposition of the Cantor phase: in the HPTed nc state the $50 \mathrm{~nm}$ grains grow to $1 \mu \mathrm{m}$ upon annealing at $700{ }^{\circ} \mathrm{C}$ and to $10 \mu \mathrm{m}$ with $800{ }^{\circ} \mathrm{C}$ for $1 \mathrm{~h}$, 
respectively. ${ }^{12}$ In contrast, such a high defect density does not exist in the sputtered film. The fact that both sputtered and HPTed samples decompose at much lower temperatures in comparison to their coarse-grained counterparts, indicates that both grain boundary energy and grain size can strongly influence the phase stability of nc CCM. The sputtered film is assumed to have lower grain boundary energy and smaller grain size. In contrast, the HPTed sample has higher grain boundary energy and larger grains. Therefore, it is not straightforward to clarify the effect of grain boundary property and grain size on the phase stability of CCM. For this purpose, comparative investigations need to be made for materials with the same grain sizes but synthesized differently. This task is beyond the scope of the current work, and will be addressed in future works.

To show the feasibility of CPP for oxidation studies, a separately coated and vacuum-annealed CPP (3 at\% Mnenriched, annealed in vacuum at $100{ }^{\circ} \mathrm{C}$ for $2.5 \mathrm{~h}$ and $150{ }^{\circ} \mathrm{C}$ for $5 \mathrm{~h}$, no decomposition observed by APT) was additionally annealed in air at $500{ }^{\circ} \mathrm{C}$ for 5 minutes (Fig. S3c, ESI $\dagger$ ). APT results in Fig. 5 illustrate the preferential oxidation of $\mathrm{Mn}$ and $\mathrm{Cr}$. An outer layer of $\mathrm{Mn}$ oxide and a sub-layer of $\mathrm{Cr}$ oxide are observed on the initial Cantor phase thin film, in agreement with literature on bulk sample oxidation. ${ }^{17}$ The $\mathrm{Mn}^{-}$and Cr-oxides on the surface of the film promote the formation of a FeCo-rich phase beneath.

To better assess phase evolution and decomposition pathways in CCMs a lower dimensional representation of the highdimensional composition data is needed. ${ }^{18}$ We propose to use multidimensional scaling (MDS) to visualize the quinary and senary composition data from the decomposition and oxidation experiments in 2D and 3D scatter plots. MDS is briefly
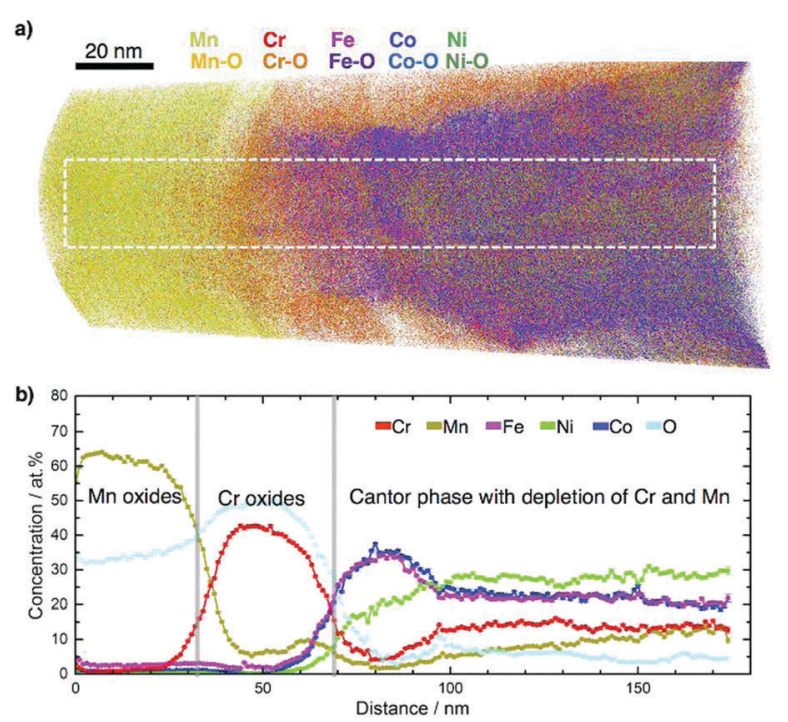

Fig. 5 Oxidation study of $\mathrm{CrMnFeCoNi}$ using CPP. Oxidation at $500{ }^{\circ} \mathrm{C}$ for $5 \mathrm{~min}$ in air leads to formation of an outer layer of $\mathrm{Mn}$ oxide and sub-layer of $\mathrm{Cr}$ oxide on the initial thin film. (a) Atom maps of the detected elements. (b) 1D concentration profiles of the elements after dissociation of all detected molecular ions (see Fig. S8, ESI $\dagger$ for distributions of alloy elements, their oxide species and concentrations) for the selected region of $20 \times 23 \times 175 \mathrm{~nm}^{3}$ indicated in (a).
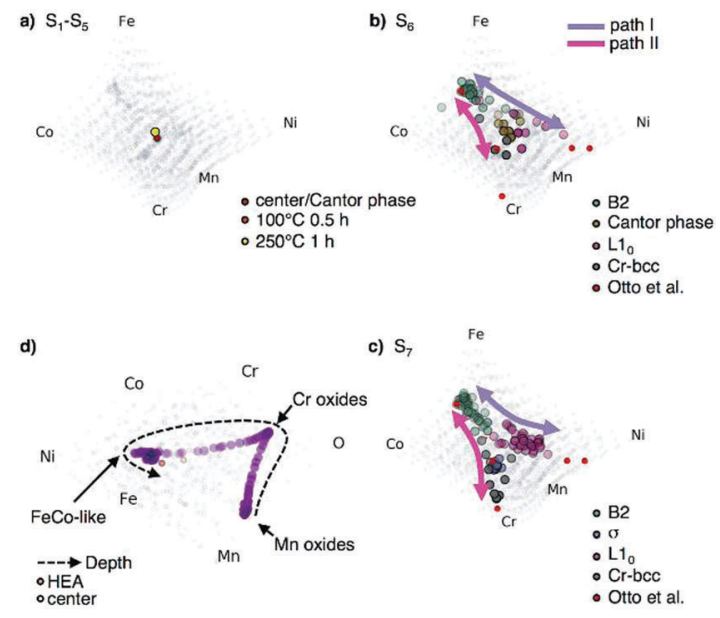

Fig. 6 MDS visualizations of phase evolution of $\mathrm{CrMnFeCoNi(O)}$ for different processing states. Results from CPP-APT are shown using a MDS visualization for (a) the states $S_{1}$ and $S_{5}$ (b) phase decomposition in state $S_{6}$ and $(c)$ in state $S_{7}$, showing the phase decomposition in comparison with bulk data from ref. 11. Colored lines indicate decomposition paths, with alternate view angles in Fig. S10, ESI†. (d) MDS visualization of the senary composition space of the oxidized CPP.

discussed in the $\mathrm{ESI} \dagger$ together with alternative visualizations. Fig. 6 shows the quinary composition data of different states which helps to track and understand phase evolution on the CPP (see video in the ESI $\dagger$ ). In processing states $S_{1}-S_{5}$ the film remains single-phase and the APT-derived compositions are close to the center (Fig. $6 \mathrm{a}$ ). In $\mathrm{S}_{6}$ and $\mathrm{S}_{7}$ decomposition occurs and its compositional development becomes directly traceable in the visualizations (Fig. $6 \mathrm{~b}$ and $\mathrm{c}$ ). The visualization of $\mathrm{S}_{6}$ shows that most of the decomposed phases appear compositionally close to the decomposition phases found by Otto et al. ${ }^{11} \mathrm{~A}$ decomposition path connects the phases $\mathrm{B} 2$ and $\mathrm{L} 1_{0}$ via the Cantor phase (purple line in Fig. 6b). This decomposition path is stabilized in $\mathrm{S}_{7}$ and a second decomposition path (magenta line in Fig. 6b and c) emerges, connecting the phases B2 and Cr-bcc via the $\sigma$-phase. These decomposition paths are not directly obtainable from assessing only the raw compositional data, indicating how MDS visualization aids the understanding of phase evolution in CCM. The decomposition path of the oxidation experiment in Fig. 6d shows the reaction of the Cantor phase into Cr- and Mn-oxides with the Cr-oxide having the higher O-content. The compositional data points in the visualization of the oxidation experiment (Fig. 6d) which lie between the fully oxidized phases are due to the transition between the phases.

\section{Conclusions}

It is shown that combinatorial processing platforms (CPP) improve substantially the efficiency of studying phase evolution and oxidation in compositionally complex materials. CPPs enable direct deposition of identical nanocrystalline films on arrays of nanoscale tips, followed by direct atomic-scale characterization of their thermal stability and oxidation resistance with APT and 
TEM. The created nanovolumes facilitate rapid phase decomposition, even at low temperatures due to short diffusion paths and without artificial introduction of high defect densities, and the CPP allows tracking phase formation and decomposition pathways in dependence on processing conditions. As a proof of concept, a 5 principle element alloy deposited on a CPP showed the same phase constitutions after several hours annealing as those observed after hundreds of days annealing time for conventional, coarse-grained bulk materials; i.e. time-consuming procedures like casting, long-term annealing of bulk materials and preparation of samples for microscopies, are not necessary.

This approach is applicable for arbitrary inorganic systems, where samples of nano-volume can be deposited on commonlyused Si-substrates (either APT tip arrays or prepared by FIB cutting) for direct investigation. With the availability of the location of each individual alloying element, the significant time reduction achieved using the CPP nano-reactors is augmented by generating high-precision insights related to the sequence of phase separation at the atomic scale and multi-dimensional data visualization. This is valuable for alloy design, in particular for designing advanced compositionally complex materials, where the phase stability is a major concern.

As an outlook, deposition of a thin film materials library on a substrate which contains an array of CPPs will combine the CPP-APT approach with the efficiency of combinatorial materials science to enable exploration of complex multinary systems and to study the existence of different decomposition pathways for compositionally complex materials. The CPP approach will next be extended by deposition of diffusion couple or composition gradient films on the tip arrays. The CPP could also be utilized as a platform for electrochemical and corrosion studies.

\section{Conflicts of interest}

There are no conflicts to declare.

\section{Acknowledgements}

The authors thank the following persons: E. George for initial discussion about investigating the stability of the Cantor alloy, S. Thienhaus for temperature-dependent resistivity measurements, R. Drautz for proof-reading the manuscript and fruitful discussions. The ZGH is acknowledged for using its facilities.
A. S. and A. L. acknowledge funding from the AHEAD project (DFG/ANR, LU 1175/22-1).

\section{Notes and references}

1 Z. Li, K. G. Pradeep, Y. Deng, D. Raabe and C. C. Tasan, Nature, 2016, 534, 227-230.

2 B. Gludovatz, A. Hohenwarter, K. V. S. Thurston, H. Bei, Z. Wu, E. P. George and R. O. Ritchie, Nat. Commun., 2016, 7, 10602.

3 B. Gludovatz, A. Hohenwarter, D. Catoor, E. H. Chang, E. P. George and R. O. Ritchie, Science, 2017, 345, 1153-1158.

4 S. Curtarolo, G. L. W. Hart, M. B. Nardelli, N. Mingo, S. Sanvito and O. Levy, Nat. Mater., 2013, 12, 191-201.

5 X. D. Xiang, X. Sun, G. Briceho, Y. Lou, K. Wang, H. Chang, W. G. Wallace-Freedman, S. Chen and P. G. Schultz, Science, 1995, 268, 1738-1740.

6 D. B. Miracle and O. N. Senkov, Acta Mater., 2017, 122, 448-511.

7 G. Schmitz, C. B. Ene and C. Nowak, Acta Mater., 2009, 57, 2673-2683.

8 C. B. Ene, G. Schmitz, R. Kirchheim and A. Hütten, Acta Mater., 2005, 53, 3383-3393.

9 T. Jeske and G. Schmitz, Mater. Sci. Eng., A, 2002, 327, 101-108.

10 B. Cantor, I. T. H. Chang, P. Knight and A. J. B. Vincent, Mater. Sci. Eng., A, 2004, 375-377, 213-218.

11 F. Otto, A. Dlouhý, K. G. Pradeep, M. Kubenova, D. Raabe, G. Eggeler and E. P. George, Acta Mater., 2016, 112, 40-52.

12 B. Schuh, F. Mendez-Martin, B. Völker, E. P. George, H. Clemens, R. Pippan and A. Hohenwarter, Mechanical properties, Acta Mater., 2015, 96, 258-268.

13 R. Z. Valiev, R. K. Islamgaliev and I. V. Alexandrov, Prog. Mater. Sci., 2000, 45, 103-189.

14 B. B. Straumal, B. Baretzky, A. A. Mazilkin, F. Phillipp, O. A. Kogtenkova, M. N. Volkov and R. Z. Valiev, Acta Mater., 2004, 52, 4469-4478.

15 D. Sata, K. Ebermann, K. Eberl and J. Maier, Nature, 2000, 408, 946-949.

16 R. Würschum, S. Herth and U. Brossmann, Adv. Eng. Mater., 2003, 5, 365-372.

17 G. Laplanche, U. F. Volkert, G. Eggeler and E. P. George, Oxid. Met., 2016, 85, 629-645.

18 C. Suh, S. C. Sieg, M. J. Heying, J. H. Oliver, W. Maier and K. Rajan, J. Comb. Chem., 2009, 11(3), 385-392. 